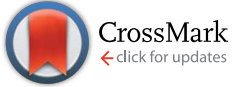

Cite this: Chem. Sci., 2017, 8, 1977

Received 22nd August 2016 Accepted 14th November 2016

DOI: $10.1039 / c 6 s c 03764 j$

www.rsc.org/chemicalscience

\section{Neutral iridium catalysts with chiral phosphine- carboxy ligands for asymmetric hydrogenation of unsaturated carboxylic acids $\uparrow$}

\begin{abstract}
Shuang Yang, ${ }^{a}$ Wen Che, ${ }^{a}$ Hui-Ling Wu, ${ }^{a}$ Shou-Fei Zhu*a and Qi-Lin Zhou ${ }^{\star a b}$
We developed neutral iridium catalysts with chiral spiro phosphine-carboxy ligands (SpiroCAP) for asymmetric hydrogenation of unsaturated carboxylic acids. Different from the cationic Crabtree-type catalysts, the iridium catalysts with chiral spiro phosphine-carboxy ligands are neutral and do not require the use of a tetrakis[3,5-bis(trifluoromethyl)phenyl]borate $\left(\mathrm{BAr}_{\mathrm{F}}{ }^{-}\right.$) counterion, which is necessary for stabilizing cationic Crabtree-type catalysts. Another advantage of the neutral iridium catalysts is that they have high stability and have a long lifetime in air. The new iridium catalysts with chiral spiro phosphine-carboxy ligands exhibit unprecedented high enantioselectivity (up to $99.4 \%$ ee) in the asymmetric hydrogenations of various unsaturated carboxylic acids, particularly for 3-alkyl-3methylenepropionic acids, which are challenging substrates for other chiral catalysts.
\end{abstract}

\section{Introduction}

Transition-metal-catalyzed asymmetric hydrogenation is one of the most useful reactions for the synthesis of optical compounds because of its perfect atom economy and operational simplicity. ${ }^{1}$ Chiral ligands are essential for this reaction. In the past decades, a tremendous number of chiral ligands have been developed for the asymmetric hydrogenation of pro-chiral unsaturated compounds. ${ }^{2}$ However, there are few chiral ligands in which oxygen is the coordinating atom for asymmetric hydrogenation of olefins. ${ }^{3} \mathrm{~A}$ few chiral phosphine-amide ligands with oxygen as the coordinating atom have been reported for rhodium- and iridium-catalyzed asymmetric hydrogenation reactions of cyclic enamides and trisubstituted olefins, respectively, with moderate to high enantioselectivity. ${ }^{\mathbf{3}, 3 \mathbf{b}}$

Recently, we developed iridium catalysts with chiral spiro phosphine-oxazoline ligands and chiral spiro aminophosphine ligands, which show high enantioselectivity for the asymmetric hydrogenation of unsaturated carboxylic acids. ${ }^{4}$ Mechanistic studies revealed that the carboxy group of the substrate anchors the molecule to the catalyst and triggers the hydrogenation reaction. On the basis of these results, we envisioned that

${ }^{a}$ State Key Laboratory and Institute of Elemento-Organic Chemistry, Nankai University, Tianjin 300071, China.E-mail: sfzhu@nankai.edu.cn; qlzhou@nankai.edu.cn ${ }^{b}$ Collaborative Innovation Center of Chemical Science and Engineering (Tianjin), Tianjin 300071, China

$\dagger$ Electronic supplementary information (ESI) available: For CIF data of $(S)$-2d, experimental procedures, and characterization data. CCDC 1498903. For ESI and crystallographic data in CIF or other electronic format see DOI: $10.1039 / \mathrm{c} 6 \mathrm{sc} 03764 \mathrm{j}$ iridium complexes with a chiral spiro phosphine-carboxy ligand might efficiently catalyze the asymmetric hydrogenation of unsaturated carboxylic acids. Carboxy groups can be introduced into chiral ligands to tune the electronic and steric properties of catalysts and thus offer new opportunities to achieve asymmetric hydrogenation of challenging substrates. Herein, we report the preparation of iridium complexes with chiral spiro phosphine-carboxy ligands (2, Scheme 1) and the use of the complexes for the hydrogenation of unsaturated carboxylic acids. These neutral iridium catalysts have several advantages: (1) they do not require the use of a tetrakis[3,5-bis(trifluoromethyl)phenyl] borate $\left(\mathrm{BAr}_{\mathrm{F}}{ }^{-}\right.$, has a molecular weight of 863) counterion, which is necessary for stabilizing chiral cationic Crabtree-type catalysts (also called Pfaltz catalysts); ${ }^{5}$ (2) they have high stability and have a long lifetime in air; and (3) they exhibit unprecedented high enantioselectivity (up to $99.4 \%$ ee) in the asymmetric hydrogenation of various unsaturated carboxylic acids, particularly for 3-alkyl-3-methylenepropionic acids, which are challenging substrates.

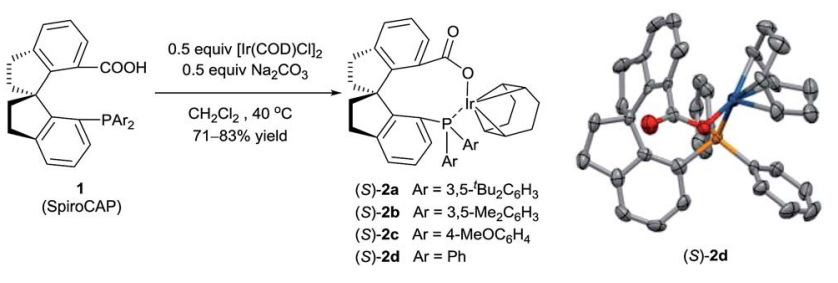

Scheme 1 Synthesis of iridium complexes of chiral spiro phosphinecarboxy ligands. The crystal structure of $(S)-2 d$ (hydrogen atoms omitted for clarity). Selected bond lengths $(\AA)$ and angles $\left(^{\circ}\right)$ for $(S)-2 d$ : Ir-O 2.085(4), Ir-P 2.3516(17); O-Ir-P 90.30(15). 


\section{Results and discussion}

Iridium complexes 2 were readily prepared with high yields (71-83\%) by refluxing a mixture of $[\operatorname{Ir}(\mathrm{COD}) \mathrm{Cl}]_{2}$, chiral spiro phosphine-carboxy ligands 1 (SpiroCAP), ${ }^{6}$ and $\mathrm{Na}_{2} \mathrm{CO}_{3}$ in $\mathrm{CH}_{2} \mathrm{Cl}_{2}$ for $1 \mathrm{~h}$ (Scheme 1). These neutral complexes were very stable; they can be purified by silica gel column chromatography and stored in air for a few months. The structure of $(S)$-2d was confirmed by X-ray diffraction analysis of a single crystal (Scheme 1), which revealed that $(S)$-1d coordinates to the iridium atom as an anionic ligand. ${ }^{7}$

Asymmetric hydrogenation of 3-alkyl-3-methylene-carboxylic acids 5 is a useful reaction because the products, chiral 3-alkyl3-methylpropionic acids, are building blocks for biologically active compounds. ${ }^{8}$ However, there are no well-established methods for the asymmetric hydrogenation of 3-alkyl-3-methylene-carboxylic acids. Uchida et al. ${ }^{9}$ and Zhang et al. ${ }^{10}$ investigated this reaction by using Ru/BINAP and Rh/DuanPhos as catalysts, respectively, but achieved only moderate enantioselectivity (up to $74 \%$ ee). Alternatively, Pfaltz et al. ${ }^{11}$ reported a hydrogenation of $\alpha, \beta$-unsaturated esters giving 3 -alkyl-3methylpropionate esters in good enantioselectivities (up to

Table 1 Iridium-catalyzed asymmetric hydrogenation of 3-methyleneheptanoic acid $^{a}$

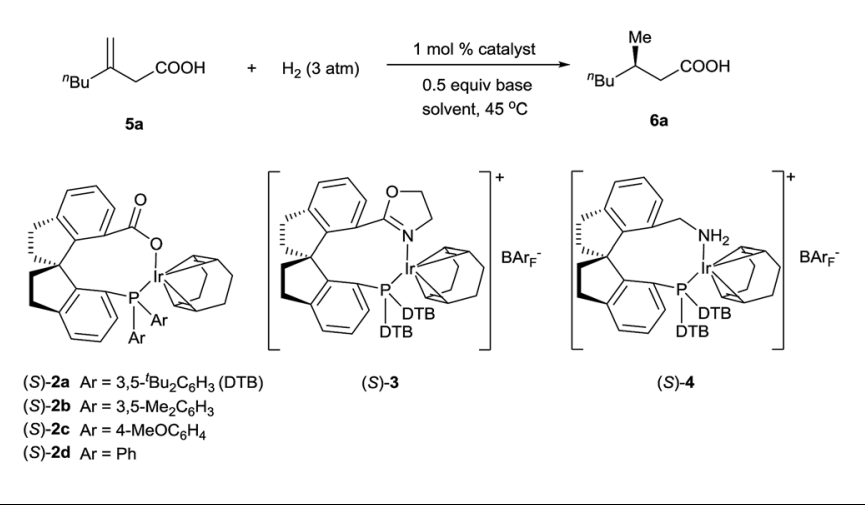

\begin{tabular}{|c|c|c|c|c|c|c|}
\hline Entry & Catalyst & Base & Solvent & Time (h) & Conv. $^{b}(\%)$ & $\mathrm{ee}^{c}(\%)$ \\
\hline 1 & $(S)-\mathbf{2 a}$ & $\mathrm{Cs}_{2} \mathrm{CO}_{3}$ & $\mathrm{MeOH}$ & 0.5 & 100 & 82 \\
\hline 2 & $(S)-\mathbf{2} \mathbf{b}$ & $\mathrm{Cs}_{2} \mathrm{CO}_{3}$ & $\mathrm{MeOH}$ & 0.5 & 100 & 83 \\
\hline 3 & $(S)-2 \mathbf{c}$ & $\mathrm{Cs}_{2} \mathrm{CO}_{3}$ & $\mathrm{MeOH}$ & 0.5 & 100 & 83 \\
\hline 4 & $(S)-2 \mathbf{d}$ & $\mathrm{Cs}_{2} \mathrm{CO}_{3}$ & $\mathrm{MeOH}$ & 0.5 & 100 & 84 \\
\hline 5 & $(S)-3$ & $\mathrm{Cs}_{2} \mathrm{CO}_{3}$ & $\mathrm{MeOH}$ & 2 & 100 & 35 \\
\hline 6 & $(S)-\mathbf{4}$ & $\mathrm{Cs}_{2} \mathrm{CO}_{3}$ & $\mathrm{MeOH}$ & 0.5 & 100 & 66 \\
\hline 7 & $(S)-2 d$ & $\mathrm{~K}_{2} \mathrm{CO}_{3}$ & $\mathrm{MeOH}$ & 0.5 & 100 & 78 \\
\hline 8 & $(S)-2 d$ & $\mathrm{Na}_{2} \mathrm{CO}_{3}$ & $\mathrm{MeOH}$ & 0.5 & 100 & 55 \\
\hline $9^{d}$ & $(S)-2 d$ & $\mathrm{NEt}_{3}$ & $\mathrm{MeOH}$ & 0.5 & 100 & 76 \\
\hline 10 & $(S)-\mathbf{2 d}$ & None & $\mathrm{MeOH}$ & 24 & 27 & 70 \\
\hline $11^{e}$ & $(S)-2 d$ & $\mathrm{Cs}_{2} \mathrm{CO}_{3}$ & $\mathrm{MeOH}$ & 0.5 & 100 & 88 \\
\hline $12^{e}$ & $(S)-2 \mathbf{d}$ & $\mathrm{Cs}_{2} \mathrm{CO}_{3}$ & ${ }^{n} \mathrm{PrOH}$ & 0.5 & 100 & 90 \\
\hline $13^{e}$ & $(S)-2 d$ & $\mathrm{Cs}_{2} \mathrm{CO}_{3}$ & ${ }^{\mathrm{i}} \mathrm{PrOH}$ & 0.5 & 100 & 91 \\
\hline $14^{e}$ & $(S)-2 d$ & $\mathrm{Cs}_{2} \mathrm{CO}_{3}$ & ${ }^{n} \mathrm{BuOH}$ & 0.5 & 100 & 93 \\
\hline $15^{e}$ & $(S)-\mathbf{2 d}$ & $\mathrm{Cs}_{2} \mathrm{CO}_{3}$ & ${ }^{t} \mathrm{BuOH}$ & 0.5 & 100 & 93 \\
\hline
\end{tabular}

${ }^{a}$ Reaction conditions: $5 \mathrm{a} /$ catalyst $/$ base $=0.5: 0.005: 0.25(\mathrm{mmol}), 2 \mathrm{~mL}$ solvent, $45{ }^{\circ} \mathrm{C} .{ }^{b}$ Determined by ${ }^{1} \mathrm{H}$ NMR. ${ }^{c}$ Determined by HPLC using a Chiralpak AD-H column. ${ }^{d} 1.0$ equiv of base was used. ${ }^{e}$ Performed at $65{ }^{\circ} \mathrm{C}$.
$94 \%$ ee). We studied the asymmetric hydrogenation of 3-methyleneheptanoic acid (5a) with iridium complexes 2 as catalysts (Table 1). When the reaction was performed with $(S)$-2a in the presence of base $\mathrm{Cs}_{2} \mathrm{CO}_{3}$ under $3 \mathrm{~atm}$ of hydrogen in methanol at $45{ }^{\circ} \mathrm{C}$, the desired product, 3-methylheptanoic acid (6a), was obtained in $82 \%$ ee (entry 1). Catalysts with less bulky $P$-aryl groups, such as 3,5-dimethylphenyl (2b), 4-methoxylphenyl (2c), and phenyl (2d), exhibited slightly higher enantioselectivities (entries 2-4). In contrast, catalysts $(S)$-3 (ref. 4c) and $(S)$-4 (ref. $4 f$ ) with chiral spiro P,N ligands gave much lower enantioselectivities (35\% and 66\% ee, respectively; entries 5 and 6). Other bases $\left(\mathrm{K}_{2} \mathrm{CO}_{3}, \mathrm{Na}_{2} \mathrm{CO}_{3}\right.$, and $\left.\mathrm{NEt}_{3}\right)$ gave lower enantioselectivities (entries 7-9), and omission of the base resulted in a poor conversion of the substrate (entry 10). Performing the reaction at $65{ }^{\circ} \mathrm{C}$ slightly increased the enantioselectivity (entry 11). The use of higher-molecular-weight alcohols as solvents led to higher enantioselectivities (entries 12-15), and ${ }^{n} \mathrm{BuOH}$ and ${ }^{t} \mathrm{BuOH}$ proved to be the most suitable solvents, giving the desired product in $93 \%$ ee. When the carboxy group of the ligand 1a was replaced by a methyl ester, only racemic product was obtained under identical reaction conditions, which clearly demonstrates that the coordination of carboxyl group of ligand to the metal of catalyst is necessary for enantiocontrol of reaction.

Under the optimal reaction conditions (Table 1, entry 14), various 3-alkyl-3-methylenepropionic acids (5a-5j) were hydrogenated in the presence of $1 \mathrm{~mol} \%$ catalyst $(S)$-2d (Table 2). All the tested substrates underwent hydrogenation within $0.5 \mathrm{~h}$ to afford the desired chiral 3-methyl-carboxylic acids with good to excellent enantioselectivity (86-98\% ee). The steric bulk of the $\mathrm{R}$ group of the substrate had a remarkable influence on the enantioselectivity; bulky alkyl groups favored high enantioselectivity $(\mathbf{6 d - 6 g})$. The hydrogenation of $\mathbf{5 j}$, which has two $\mathrm{C}=\mathrm{C}$

Table 2 Asymmetric hydrogenation of 3-alkyl-3-methylenepropionic acids catalyzed by $(S)-2 \mathrm{~d}^{a}$

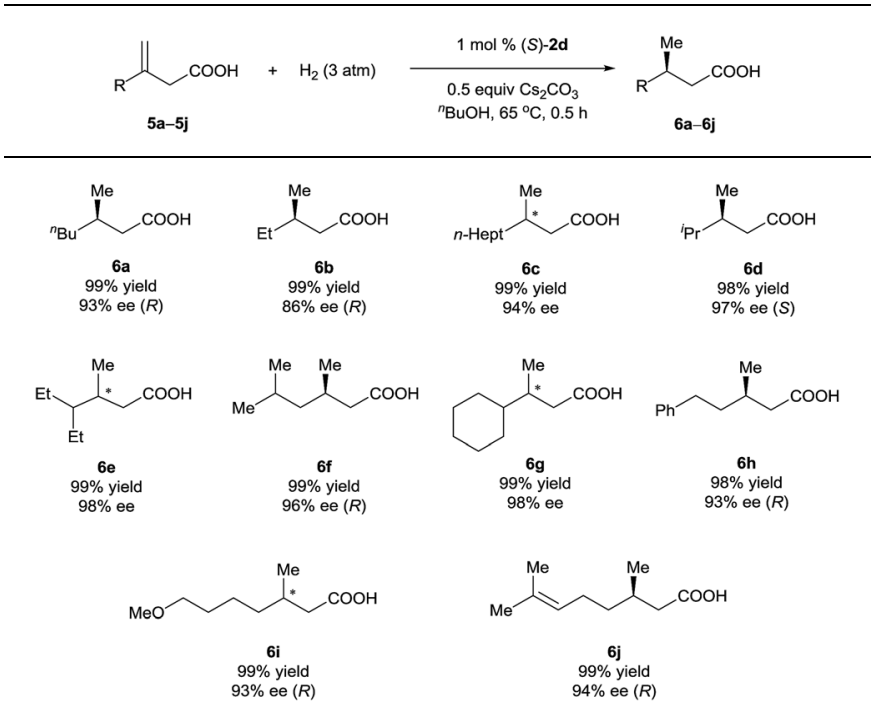

\footnotetext{
${ }^{a}$ Reaction conditions and analytical methods were the same as those described in entry 14 of Table 1 . Full conversion was obtained in all cases.
} 


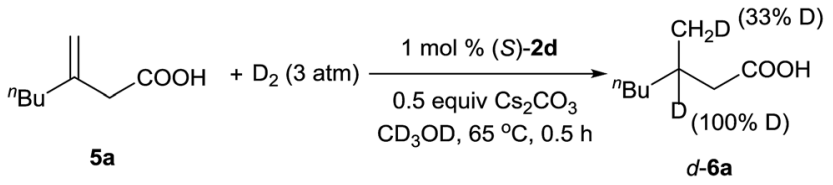

Scheme 2 Deuterium-labeling experiment.

bonds, occurred only at the $\beta$-olefin; the remote olefin moiety was untouched.

A deuterium-labeling experiment was performed to determine whether the olefin migrated during the hydrogenation reaction (Scheme 2). The absence of deuterium at the $\alpha$-position of hydrogenation product $d$-6a clearly showed that the double bond did not migrate during the reaction.

In addition to 3-alkyl-3-methylenepropionic acids, other representative unsaturated carboxylic acids, such as $\alpha$-methyl cinnamic acid (7a), tiglic acid (7b), $\alpha$-phenyl butenoic acid (7c), $\alpha$-benzyloxy cinnamic acid (7d), 2-(4-isobutylphenyl)acrylic acid (7e), and 2-methyleneheptanoic acid (7f), also underwent hydrogenation catalyzed by $(S)$-2d (Table 3), producing the corresponding chiral carboxylic acids in high yields (97-99\%) with excellent enantioselectivities (94-99.4\% ee). These results show that neutral iridium catalysts 2 are among the most efficient chiral catalysts reported to date for the asymmetric hydrogenation of unsaturated carboxylic acids. ${ }^{4 a, 9-12}$

The (S)-14-methyloctadec-1-ene is a female sex pheromone of the peach leafminer moth (Lyonetia clerkella). Several syntheses of (S)-14-methyloctadec-1-ene have been reported using enzymatic catalysis, ${ }^{13}$ chiral starting materials, ${ }^{14}$ or chiral auxiliaries. ${ }^{15}$ To demonstrate the potential application of the asymmetric hydrogenation of 3-alkyl-3-methylenepropionic acids in organic synthesis, we carried out a synthesis of $(S)$-14methyloctadec-1-ene (Scheme 3). 3-Methyleneheptanoic acid 5a, which was easily prepared in $80 \%$ yield by a ring-opening reaction of commercially available 4-methyleneoxetan-2-one with a Grignard reagent, was hydrogenated in the presence of

Table 3 Hydrogenation of other types of unsaturated carboxylic acids $^{a}$

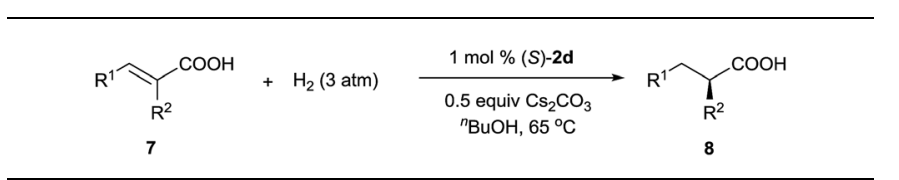

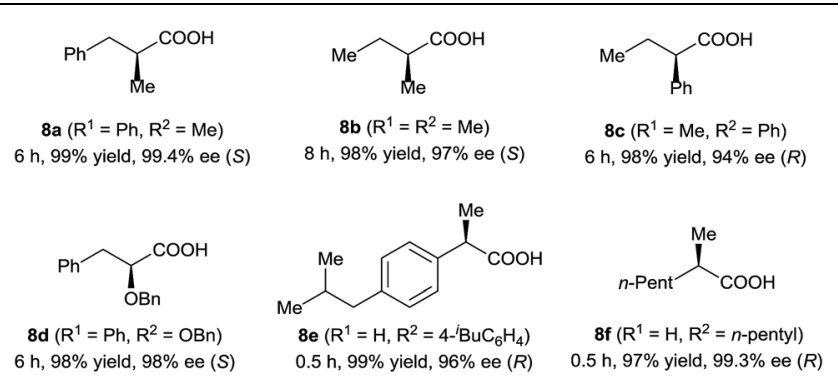

\footnotetext{
${ }^{a}$ Reaction conditions and analytical methods were the same as those described in entry 14 of Table 1 . Full conversion was obtained in all cases.
}

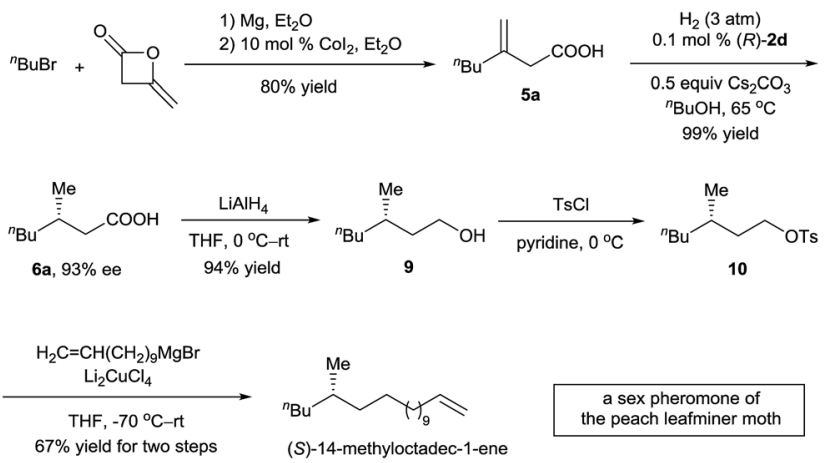

Scheme 3 Total synthesis of (S)-14-methyloctadec-1-ene.

0.1 mol\% catalyst $(R)$-2d to produce acid $(S)$-6a in $99 \%$ yield with 93\% ee. Acid $(S)-6 a$ was reduced with $\mathrm{LiAlH}_{4}$ to alcohol 9 in $94 \%$ yield. Finally, $\mathbf{9}$ was converted to $(S)$-14-methyloctadec-1-ene by tosylation and subsequent coupling with a Grignard reagent in $67 \%$ yield for the two steps. Thus, the total synthesis of (S)-14-methyloctadec-1-ene was accomplished in $50 \%$ overall yield starting from commercially available materials.

\section{Conclusions}

In summary, we developed a new type of chiral spiro phosphinecarboxy ligand, which can form iridium complexes without the need for a stabilizing counterion. Neutral iridium complexes of these ligands catalyzed the asymmetric hydrogenation of various unsaturated carboxylic acids with excellent enantioselectivity. In particular, these catalysts allowed us to achieve highly enantioselective hydrogenation of 3-alkyl-3-methylenepropionic acids, which are challenging substrates for previously reported chiral catalysts. Additional studies on the applications of these neutral iridium catalysts with chiral phosphine-carboxy ligands to other asymmetric reactions are underway in our laboratory.

\section{Acknowledgements}

We thank the National Natural Science Foundation of China, the National Basic Research Program of China (2012CB821600), the "111" project (B06005) of the Ministry of Education of China, and the National Program for Support of Top-notch Young Professionals for financial support.

\section{Notes and references}

1 For reviews, see: (a) Handbook of Homogeneous Hydrogenation, ed. J. G. de Vries and C. J. Elsevier, WileyVCH, Weinheim, Germany, 2007; (b) Asymmetric Catalysis on Industrial Scale: Challenges, Approaches and Solutions, ed. H.-U. Blaser and E. Schmidt, Wiley-VCH, Weinheim, Germany, 2004; (c) J.-H. Xie and Q.-L. Zhou, Acta Chim. Sin., 2012, 70, 1427-1438.

2 (a) W. Tang and X. Zhang, Chem. Rev., 2003, 103, 3029-3070; (b) Y.-M. He and Q.-H. Fan, Org. Biomol. Chem., 2010, 8, 
2497-2504; (c) B. Zhao, Z. Han and K. Ding, Angew. Chem., Int. Ed., 2013, 52, 4744-4788.

3 (a) J. Meeuwissen, R. J. Detz, A. J. Sandee, B. de Bruin and J. N. H. Reek, Dalton Trans., 2010, 39, 1929-1931; (b) D. Rageot, D. H. Woodmansee, B. Pugin and A. Pfaltz, Angew. Chem., Int. Ed., 2011, 50, 9598-9601; For achiral P,O ligands used in hydrogenation of olefins, see: (c) J. Cipot, R. McDonald and M. Stradiotto, Organometallics, 2006, 25, 29-31; (d) K. Park, P. O. Lagaditis, A. J. Lough and R. H. Morris, Inorg. Chem., 2013, 52, 5448-5456; For chiral $\mathrm{P}, \mathrm{O}$ ligands used in hydrogenation of ketones, see: $(e)$ F. Jiang, K. Yuan, M. Achard and C. Bruneau, Chem. Eur. J., 2013, 19, 10343-10352.

4 For reviews, see: (a) S. Khumsubdee and K. Burgess, ACS Catal., 2013, 3, 237-249; (b) T. Besset, R. Gramage-Doria and J. N. H. Reek, Angew. Chem., Int. Ed., 2013, 52, 87958797. For examples, see: (c) S. Li, S.-F. Zhu, C.-M. Zhang, S. Song and Q.-L. Zhou, J. Am. Chem. Soc., 2008, 130, 85848585; (d) S. Li, S.-F. Zhu, J.-H. Xie, S. Song, C.-M. Zhang and Q.-L. Zhou, J. Am. Chem. Soc., 2010, 132, 1172-1179; (e) S. Song, S.-F. Zhu, S. Yang, S. Li and Q.-L. Zhou, Angew. Chem., Int. Ed., 2012, 51, 2708-2711; (f) S.-F. Zhu, Y.-B. Yu, S. Li, L.-X. Wang and Q.-L. Zhou, Angew. Chem., Int. Ed., 2012, 51, 8872-8875; (g) S. Song, S.-F. Zhu, Y.-B. Yu and Q.-L. Zhou, Angew. Chem., Int. Ed., 2013, 52, 1556-1559.

5 For the initial development of chiral iridium/phosphineoxazoline complexes, see: (a) A. Lightfoot, P. Schnider and A. Pfaltz, Angew. Chem., Int. Ed., 1998, 37, 2897-2899. For reviews, see: (b) S. J. Roseblade and A. Pfaltz, Acc. Chem. Res., 2007, 40, 1402-1411; (c) J. J. Verendel, O. Pàmies, M. Diéguez and P. G. Andersson, Chem. Rev., 2014, 114, 2130-2169.

6 For the preparation of compounds 1, see: S.-F. Zhu, J.-B. Xie, Y.-Z. Zhang, S. Li and Q.-L. Zhou, J. Am. Chem. Soc., 2006, 128, 12886-12891.

7 CCDC 1498903 contains the supplementary crystallographic data for compound $(S)$-2d. See $\mathrm{ESI}_{\dagger} \dagger$ for details.

8 (a) Y. Leblanc, W. C. Black, S. S. Chan, S. Charleson, D. Delorme, D. Denis, J. Y. Gauthier, E. L. Grimm, R. Gordon, D. Guay, P. Hamel, S. Kargman, C. K. Lau, J. Mancini, M. Ouellet, D. Percival, P. Roy, K. Skorey, P. Tagari, P. Vickers, E. Wong, L. Xu and P. Prasit, Bioorg. Med. Chem. Lett., 1996, 6, 731-736; (b) H. J. Jessen, D. Barbaras, M. Hamburger and K. Gademann, Org. Lett., 2009, 11, 3446-3449.
9 M. Saburi, H. Takeuchi, M. Ogasawara, T. Tsukahara, Y. Ishii, T. Ikariya, T. Takahashi and Y. Uchida, J. Organomet. Chem., 1992, 428, 155-167.

10 X. Sun, L. Zhou, C.-J. Wang and X. Zhang, Angew. Chem., Int. Ed., 2007, 46, 2623-2626.

11 (a) S. Kaiser, S. P. Smidt and A. Pfaltz, Angew. Chem., Int. Ed., 2006, 45, 5194-5197; (b) D. H. Woodmansee, M.-A. Müller, L. Tröndlin, E. Hörmann and A. Pfaltz, Chem.-Eur. J., 2012, 18, 13780-13786.

12 For other selected examples of catalytic asymmetric hydrogenation of unsaturated carboxylic acids, see: $(a)$ T. Ohta, H. Takaya, M. Kitamura, K. Nagai and R. Noyori, J. Org. Chem., 1987, 52, 3174-3176; (b) T. Hayashi, N. Kawamura and Y. Ito, J. Am. Chem. Soc., 1987, 109, 7876-7878; (c) C.-C. Pai, C.-W. Lin, C.-C. Lin, C.-C. Chen, A. S. C. Chan and W. T. Wong, J. Am. Chem. Soc., 2000, 122, 11513-11514; (d) X. Cheng, Q. Zhang, J.-H. Xie, L.-X. Wang and Q.-L. Zhou, Angew. Chem., Int. Ed., 2005, 44, 1118-1121; (e) R. Hoen, J. A. F. Boogers, H. Bernsmann, A. J. Minnaard, A. Meetsma, T. D. Tiemersma-Wegman, A. H. M. de Vries, J. G. de Vries and B. L. Feringa, Angew. Chem., Int. Ed., 2005, 44, 42094212; (f) D. M. Tellers, J. C. McWilliams, G. Humphrey, M. Journet, L. DiMichele, J. Hinksmon, A. E. McKeown, T. Rosner, Y. Sun and R. D. Tillyer, J. Am. Chem. Soc., 2006, 128, 17063; $(g)$ W. Chen, P. J. McCormack, K. Mohammed, W. Mbafor, S. M. Roberts and J. Whittall, Angew. Chem., Int. Ed., 2007, 46, 4141-4144; (h) Y. Li, K. Dong, Z. Wang and K. Ding, Angew. Chem., Int. Ed., 2013, 52, 6748-6752; (i) X. Liu, Z. Han, Z. Wang and K. Ding, Angew. Chem., Int. Ed., 2014, 53, 1978-1982.

13 S. Sankaranarayanan, A. Sharma, B. A. Kulkani and S. Chattopadhyay, J. Org. Chem., 1995, 60, 4251-4254.

14 (a) M. Kato and K. Mori, Agric. Biol. Chem., 1985, 49, 24792480; (b) K. Mori and M. Kato, Liebigs Ann. Chem., 1985, 2083-2087; (c) R. Y. Kharisov, E. R. Latypova, R. F. Talipov, R. R. Muslukhov, G. Y. Ishmuratov and G. A. Tolstikov, Russ. Chem. Bull., 2003, 52, 2267-2269; (d) G. Y. Ishmuratov, V. A. Vydrina, I. S. Nazarov, Y. A. Galkina, M. P. Yakovleva, I. F. Lobko, R. R. Muslukhov and A. G. Tolstikov, Chem. Nat. Compd., 2013, 48, 981-984.

15 (a) E. Reyes, J. L. Vicario, L. Carrillo, D. Badía, U. Uria and A. Iza, J. Org. Chem., 2006, 71, 7763-7772; (b) T. Zhang, W.-L. Ma, T.-R. Li, J. Wu, J.-R. Wang and Z.-T. Du, Molecules, 2013, 18, 5201-5208. 